\title{
Study on the Teaching Method of Ancient Chinese in the specialty of elementary education
}

\author{
Zhang Li \\ (Department of Chinese Literature, Qilu Normal University, Jinan 250200, China)
}

Fund Project: Young Teachers Research Fund Project ofQilu Normal University "Ancient Chinese Teaching and Traditional Culture Inheritance - Taking Etiquette Culture as an Example" (Project No: 2014W1102)

Key words: normal university; primary education; ancient Chinese; teaching style

Abstract: The ancient Chinese course is one of the basic courses of primary education in normal university. Its purpose is to make students understand the basic knowledge of ancient Chinese, enhance the reading and understanding ability of ancient Chinese, and inherit and carry forward the ancient culture better through professional knowledge of ancient poetry, classical Chinese teaching, and enhance the quality of primary school teaching. At present, the reform of ancient Chinese teaching in primary school is still deepening. The traditional teaching method cannot meet the requirements of the curriculum. Only by setting up educational goals at different levels and increasing teaching content in many ways can improve the teaching of ancient Chinese and improve the teaching of ancient Chinese and teaching effectiveness.

The establishment of the ancient Chinese curriculum in the normal university is to improve the students' ability of reading comprehension and to carry out classical Chinese teaching. Although the primary education and Chinese language and literature education in the teaching requirements are significantly different, but the purpose of teaching is the same, which is to encourage students to read ancient books, to enhance the ability to carry out classical Chinese teaching. Therefore, the voice, vocabulary, cultural connotation and other teaching is particularly important [1]. Many modern Chinese vocabulary in the primary school stage still retains the meaning and use of ancient Chinese. In the face of these words, teachers should point out the common points and differences of ancient and modern usage and avoid the students' misunderstandings. Therefore, we should pay attention to the ancient Chinese curriculum of primary school students'normal education, and lay a solid foundation for students' future teaching.

\section{Build a full range of multi-level curriculum objectives}

The goal of the course is the important index of the teaching of primary school. To improve the traditional curriculum objectives, to meet the social requirements and curriculum development of 
teaching objectives: first of all, to recognize the ancient Chinese style knowledge, cultural knowledge and basic knowledge; Secondly, to master the classical method of classical Chinese teaching; Thirdly, to use linguistic theory knowledge of language materials for finishing and analysis; Finally, to find the problem and correctly handle the teaching problems. This course reflects the full range of multi-level features. The first two more emphasis on basic knowledge, the latter two are more emphasis on the use of basic knowledge. The development and implementation of the new curriculum objectives, highlighting the "basic, application, adjustment, innovation" thinking, effective transfer of students to learn the enthusiasm of classical learning, so that students can have a solid basic knowledge, but also have a strong ability to innovate, enhance the quality of teaching [2].

The primary school education specialty is to cultivate the applied and compound teacher talents for the primary school, let the students realize the learning goal and the learning motivation clearly. At the beginning of the school, students from the different aspects of vocational education, so that the "teacher" to carry out the study of ancient Chinese courses, reflecting the enthusiasm and initiative of learning. And teachers of ancient Chinese teachers to play a good triple role, the first one is the dissemination of knowledge, teachers to help students understand and master the professional knowledge; the second is training skills, in the teaching process to train students teaching skills; the third one is teaching methods guide. Through the training of students to teach the teachings of science [3]. Teachers of ancient Chinese teachers should teach the teaching of primary school students so that they can understand the contents of ancient Chinese, to teach both the basic knowledge and teaching skills and teaching methods so that students can quickly complete their primary school teaching and management work after graduation. Short employment adaptation period, improve the competitiveness of employment, to help them become qualified, excellent primary school teachers.

\section{Set up to "attach importance to basic education and professional development" as the core of the teaching content}

In the course of teaching, the ancient Chinese curriculum should mobilize students' independent analysis and innovation consciousness, enhance their ability of innovation and improve the basic knowledge level and professional ability of students from the aspects of pronunciation, vocabulary and cultural connotation.

Mr. Wang Li's textbook "Ancient Chinese" is a kind of content system which combines the common words, general theory and literary anthology, which has strong scientific and rationality. Anthology part of the collection of 265 articles, according to the current lack of specific 
circumstances clear and fine talk about the article, and primary school poetry will have a strong connection or a representative article as fine talk about the article, you can arrange 16 fine Speak, 10 talk, 30 extracurricular reading. Do not study the ancient books, only contact with people note this, it is difficult to cultivate excellent reading ability and understanding. Therefore, the extracurricular reading books to choose the white text and the ancient notes of the election, teachers in the students under the premise of independent understanding and analysis of the typical issues to focus on both the students to understand the original appearance of ancient books, but also guide students from learning knowledge to cultivate the direction of capacity [4]. In this learning process, teachers to develop project tasks, completed by the students' sub-groups to encourage students to explain each other to explain, improve understanding and professional skills, let it with the task of learning and harvest.

To explain the basic knowledge, so that students of ancient Chinese knowledge from the perceptual knowledge to rational knowledge, and found that the language contained in the law, can be analyzed from the following parts. The first one is the text part. Introduce the changes of Chinese characters, structure, from the original meaning of the shape analysis, the application in ancient books and other issues. In explaining the Chinese characters and structure, in order not to repeat the text, modern Chinese teaching content, only to explain the basic knowledge. The second one is the vocabulary part, to explain the ancient and modern words of the same point and the difference between the polysyllabic and monosyllabic words, the meaning of the word and its original meaning, to identify synonyms and so on. At the same time to explain the ancient Chinese common words, students master the common words more than a thousand, in reading can basically understand the contents of ancient books. The third one is the grammar part, explain the dialect words, passive sentences and judgments sentence, word order, word class and so on. It is argued that the grammatical system should be based on the specific situation of primary school language teaching, and establish the ancient Chinese curriculum system of normal university system which is compatible with the primary school language system. The fourth one is phonological part, including the ancient phonetic common sense of ancient Chinese language reading of the application. This part of the content is more abstract, and read the ancient text is not close, direct relationship, in the choice of teaching content, only need to explain to the students the basic knowledge of the phonology.

\section{Innovative teaching methods to enhance students' professionalism}

In the course of carrying out the teaching of ancient Chinese, special attention is paid to the selection of teaching methods, so as to cultivate students' learning ability, ability to deal with 
problems, innovation ability, research ability and professional ability, and to innovate and reform teaching methods.

At present, there are few courseware related to ancient Chinese teaching, and the application of computer technology in teaching can enhance the teaching quality, analyze teaching difficulties and key contents, and improve students' interest in learning and learning effectiveness [5]. In addition, you can significantly reduce the teaching time, easy to learn the purpose of learning. The ancient Chinese courseware of primary school education specialty design reflects three characteristics: first, the content is rich and highly systematic. Courseware involves grammar, vocabulary, text and other important content. To study the content, learning methods, learning difficulties and focus, learning requirements, learning objectives as the backbone, reflecting the strong context and level. In the learning content part, can show different units of learning methods, learning difficulties and focus, knowledge points. In this way, even if there is no teacher guidance, students can also self-study, improve student efficiency. Second, the operation is simple and convenient. In the operator interface in various departments, each chapter between any search, jump, even if the operator does not use the computer experience and technology, you can follow the prompts, quick use of courseware. Finally, the courseware production intuitive and interesting, there are a variety of linguistic works cover and other books, there are regular script, membership, Xiaozhuan, Oracle and other pictures, ancient painting and calligraphy, classical folk music, improve student operation and learning interest.

In the development of ancient Chinese teaching, teachers should be vivid about the language and language phenomenon, the use of comparative analysis, so that students clearly understand the ancient and modern Chinese in the grammar, vocabulary, voice and other aspects of understanding the ancient Chinese connotation and characteristics. Comparison of the more frequent use of ancient and modern comparison and vertical comparison of ancient Chinese and other two methods. The reform of this teaching method, science and effective to make up for the current stage of ancient Chinese to pay attention to explain the basic knowledge, practical training less, different analysis and other issues. In addition, comparative analysis for students can expand their thinking, increase the knowledge, mobilize the curiosity, the formation of critical thinking, the establishment of professional quality are particularly special role and significance.

In the teaching of ancient Chinese, teachers should pay attention to enhance students' practical ability and creative spirit, use the set doubt, organize discussion, guide the coaching, summed up the students, students and students interactive teaching method to support students to think, diligent questions, The subjective initiative. For example, in the study of ancient Chinese judgment sentence, the ancient Chinese sentence enumeration part of the students to how to distinguish between ancient 
and modern Chinese judge the difference between the judge, to encourage students to analyze and discuss, let it be in the comparison to understand the characteristics of the sentence The basic form, and then the teacher to correct the results of the analysis, supplement, summary. In the teaching, the students to ask questions, some lack of analysis based on some of the unique insight, teachers to guide, encourage, let students analyze and discuss, use spare time to find literature, personal views and ideas written papers, not only to develop students Brave suspicion of the spirit, but also enhance the students' in-depth exploration of professionalism and speculative ability. With the interactive teaching model, so that students more solid grasp of basic knowledge, will be esoteric, boring ancient Chinese turned more interesting and vivid. According to the survey, the use of this method to more than $90 \%$ of students interested in in-depth study of ancient Chinese.

Normal school education in primary school to carry out ancient Chinese teaching, according to primary school language teaching and student practice, to the purpose of teaching as the focus, innovative teaching content and teaching methods. By reading the "ancient Chinese" teaching materials to accumulate language knowledge, so that students have a sense of ancient Chinese perception; master the basic knowledge, understand the ancient Chinese law. At the same time, it is necessary to combine computer technology with ancient Chinese knowledge, use comparative analysis, interactive teaching methods, develop a variety of job training and other methods, both to improve students' enthusiasm for learning, but also to cultivate their professional quality, enhance the quality of ancient Chinese teaching At the same time, so that the professional curriculum is full of vitality and vitality.

\section{References:}

[1] Zhang Zuodong. Elementary School Education Professional Chinese Literature Content of the Relevance of Thinking [J]. Hechi University, 2016, (06): 102-105.

[2] Zhang Shuqin. On the Cultural Inheritance of Ancient Chinese Teaching in Teachers' Colleges [J].Journal of Ningbo Institute of Education, 2011, (01): 45-48.

[3] Peng Xiaohui. Primary School Education Undergraduate Professional Modern Chinese Curriculum Teaching Status Quo and Countermeasures [J]. Journal of Hunan First Teachers College, 2014, (02): 16-19.

[4] Bian Chunli. Problems and Countermeasures of Modern Chinese Teaching in Primary Education Specialty [J]. Education Exploration, 2014, (06): 47-48.

[5] Yang Huidong. Chinese International Education Major Ancient Chinese Teaching Reform [J]. Shaanxi Education (Higher Education), 2014, (12): 62-63. 